\title{
DYNAMICAL FRICTION IN THE COMA CLUSTER
}

\author{
M. LECAR \\ Center for Astrophysics, Harvard College Observatory and Smithsonian Astrophysical Observatory, \\ Cambridge, Mass., U.S.A.
}

\begin{abstract}
If the galaxies composing the Coma Cluster were initially 'Ostriker-Peebles' galaxies (i.e., isothermal spheres extending to $250-500 \mathrm{kpc}$ ), the hidden mass is likely to be tidally torn-off halos. Owing to dynamical friction, the remnant cores tend to spiral in to the center of the cluster. This happens as long as the hidden mass, whatever its origin, consists of objects much less massive than galaxies.
\end{abstract}

Ostriker and Peebles (1973) suggested that, in order to be stable against bar formation (see also Bardeen, 1974), galaxies should have most of their mass in extended halos. An isothermal sphere density profile would also explain the observed flat rotation curves (Roberts, 1974), which requires that their masses increase linearly with their radii. For illustration, I will assume that an 'Ostriker-Pebbles' galaxy can be characterized by the relation

$$
M=10^{10} M_{\odot} \times r(\mathrm{kpc}) .
$$

For an isothermal sphere, $M \cong\left(2 c^{2} / 3 G\right) \times r$, as $r$ becomes large. The above relation (1) results from taking the internal dispersion velocity, $c=254 \mathrm{~km} \mathrm{~s}^{-1}$.

Studies by Omer et al. (1965), Peebles (1970), Rood et al. (1972), Bahcall (1972), and Oemler (1973) provide a relatively consistent (to within a factor of 2) set of parameters for the Coma Cluster. Their results (adjusted to $H=50 \mathrm{~km} \mathrm{~s}^{-1} \mathrm{Mpc}^{-1}$ ) can be modeled by assuming Coma to be distributed like an isothermal sphere with

$$
\begin{aligned}
M & =2 \times 10^{15} M_{\odot}, \\
R & =4 \mathrm{Mpc}, \\
c & =1800 \mathrm{~km} \mathrm{~s}^{-1}, \\
r_{c} & =0.25 \mathrm{Mpc}, \\
\varrho_{0} & =3 \times 10^{-3} M_{\odot} \mathrm{pc}^{-3} .
\end{aligned}
$$

The core radius, $r_{c}$, is defined as that radius where the projected density falls to half its central value, and is equal to three isothermal structure lengths. The isothermal structure length, $a$, is related to the dispersion velocity, $c$, and the central density, $\varrho_{0}$, by

$$
a^{2}=\frac{c^{2}}{12 \pi G \varrho_{0}}=\left(\frac{r_{c}}{3}\right)^{2} .
$$

I will assume that the galaxies initially had masses of $2.5 \times 10^{12} M_{\odot}$ and radii of 250 $\mathrm{kpc}$. If each galaxy in the cluster occupies a volume $4 \pi r^{3} / 3$, then the mean value of $r$ is

$$
\bar{r}=N^{-1 / 3} R \text {. }
$$

By taking $N=800$ and $R=4 \mathrm{Mpc}, \bar{r}=430 \mathrm{kpc}$. At $1 \mathrm{Mpc}$ from the center, $r=240$ 
$\mathrm{kpc}$, decreasing to $60 \mathrm{kpc}$ at the center. Thus, interior to $1 \mathrm{Mpc}$, the initial galaxies overlap, and it seems likely that they would lose their halos through tidal interactions with their neighbors. Alladin $(1965,1974)$, Alladin et al. (1974), Sastry and Alladin (1970), Gallagher and Ostriker (1972), Lauberts (1974), and Biermann (1974) have studied penetrating collisions between spherical galaxies. Biermann found, for conditions similar to what I have assumed for the Coma Cluster (relative velocities between the galaxies about 7 times their internal dispersion velocities), that for encounter distances $\frac{1}{4}$ of the radius of the galaxy, as much as $\frac{1}{4}$ of the mass could be dispersed. However, as the binding energy of the galaxy (characterized by $\left(254 \mathrm{~km} \mathrm{~s}^{-1}\right)^{2}$ per unit mass) is less than $3 \%$ of its kinetic energy of center of mass motion (characterized by $\left(1800 \mathrm{~km} \mathrm{~s}^{-1}\right)^{2}$ per unit mass), the galaxies should hardly slow down as they shed their halos.

I now turn to the orbits of the remnant cores swimming in a sea of halo material. If the sea consists of objects (e.g., $M$ dwarfs) whose mass is much less than that of a galaxy, Spitzer (1969) and Saslaw and de Young (1971) have shown that the galaxies will tend to settle to the center of the cluster. Locally, the galaxies try to reach equipartition with the stars, and since the galaxies have too much kinetic energy, they slow down. But, in a gravitational field, to slow down means to fall, and to fall means to speed up. The galaxies can not attain equipartition, but they keep trying. A less picturesque description is given by 'dynamical friction'. The following formula for the rate of change of kinetic energy per unit mass is adapted from Hénon (1973),

$$
\frac{\mathrm{d}}{\mathrm{d} t}\left(\frac{1}{2} V^{2}\right)=-\frac{2 \pi G \varrho \cdot G M \ln \Lambda}{V},
$$

where $V$ is the velocity of the galaxy, $M$ is its mass, $\varrho$ is the density of field stars, and $\Lambda$ can be taken as $M_{\text {cluster }} / M=800$.

I have assumed, in the above, that the galaxies have the same velocity dispersion as the field stars and that the fraction with rms velocity less than the mean is $\frac{1}{2}$. As only those field stars with velocities less than that of the galaxy contribute to dynamical friction, the process quenches quickly if the field stars heat up relative to the galaxies. The process becomes 10 times less efficient if the field stars are 3 times faster (rms) than the galaxies.

It is convenient to adopt the dimensionless variables appropriate for an isothermal sphere. If we let

$$
\begin{aligned}
& V=c \cdot v \\
& \varrho=\varrho_{0} \cdot \delta \\
& t=\omega^{-1} \cdot \tau, \quad \omega=c / a=\sqrt{\left(12 \pi G \varrho_{0}\right)},
\end{aligned}
$$

Equation (5) can be written

$$
\frac{\mathrm{d}}{\mathrm{d} \tau}\left(\frac{1}{2} v^{2}\right)=-\left\{\frac{1}{6}\left[\frac{\left(G M / c^{2}\right)}{a}\right] \ln \Lambda\right\} \cdot \frac{\delta}{v} .
$$


As the galaxy loses kinetic energy, it becomes more tightly bound to the cluster. Consider a galaxy in a circular orbit with velocity $V$. Its orbit is described by

$$
\begin{aligned}
V^{2} & =\frac{G M(r)}{r}, \\
E & =\frac{1}{2} V^{2}+\int_{0}^{r} \frac{G M(r)}{r^{2}} \mathrm{~d} r=\frac{1}{2} \frac{G M(r)}{r}+\int_{0}^{r} \frac{G M(r)}{r^{2}} \mathrm{~d} r, \\
\frac{\mathrm{d} E}{\mathrm{~d} r} & =\frac{1}{2}\left[\frac{G M(r)}{r^{2}}+4 \pi G \varrho r\right] .
\end{aligned}
$$

In isothermal units,

$$
\begin{aligned}
r & =a x, \\
M(x) & =4 \pi \varrho_{0} a^{3} \mu(x), \quad \mu(x)=\int_{0}^{x} \delta(x) x^{2} \mathrm{~d} x, \\
c^{2} & =12 \pi G \varrho_{0} a^{2}, \\
E & =c^{2} \varepsilon,
\end{aligned}
$$

and Equation (8) is written

$$
\begin{aligned}
v^{2} & =\frac{1}{3} \frac{\mu(x)}{x}, \\
\varepsilon & =\frac{1}{6} \frac{\mu(x)}{x}+\frac{1}{3} \int_{0}^{x} \frac{\mu(x)}{x^{2}} \mathrm{~d} x, \\
\frac{\mathrm{d} \varepsilon}{\mathrm{d} x} & =\frac{1}{6}\left[x \delta(x)+\frac{\mu(x)}{x}\right] .
\end{aligned}
$$

Combining Equations (7) and (9) yields

$$
\frac{\mathrm{d} x}{\mathrm{~d} \tau}=\frac{\mathrm{d} x}{\mathrm{~d} \varepsilon} \cdot \frac{\mathrm{d} \varepsilon}{\mathrm{d} \tau}=\frac{-K}{x \sqrt{\mu(x) / x}\left\{1+\left[\mu(x) / x^{3} \delta(x)\right]\right\}} \equiv \frac{-K}{x \Phi(x)},
$$

where $K=\sqrt{3}\left[\left(G M / c^{2}\right) / a\right] \ln \Lambda$. The quantity $K^{-1}=65 \times a(\mathrm{Mpc})$ and the unit of time is $a / c=5.4 \times 10^{8} \mathrm{yr} \times a(\mathrm{Mpc})$. As $x \rightarrow 0, \delta \rightarrow 1, \mu \rightarrow x^{3} / 3$, and $\Phi \rightarrow 4 x / 3 \sqrt{3}$.

In this limit, Equation (10) integrates to

$$
\left(\frac{x}{x_{0}}\right)^{3}=1-\frac{9 \sqrt{3} K}{4} \frac{\tau}{x_{0}^{3}}
$$

and $\left(x / x_{0}\right)=\frac{1}{2}$ when $\tau=(7 / 18 \sqrt{3})\left(x_{0}^{3} / K\right)$. Since $t=a / c, t_{1 / 2}=8 \times 10^{9} \mathrm{yr} \times r^{3}(\mathrm{Mpc}) /$ $a(\mathrm{Mpc})$. As $x \rightarrow \infty, \delta \rightarrow 2 / x^{2}, \mu \rightarrow 2 x$, and $\Phi \rightarrow 2 \sqrt{2}$. 
In this limit, Equation (10) integrates to

$$
\left(\frac{x}{x_{0}}\right)^{2}=1-\frac{K}{\sqrt{2}} \frac{\tau}{x_{0}^{2}},
$$

and $\left(x / x_{0}\right)=\frac{1}{2}$ when $\tau=(3 / 2 \sqrt{2})\left(x_{0}^{2} / K\right)$. The quantity $t_{1 / 2}=3.7 \times 10^{10} \mathrm{yr} \times r^{2}(\mathrm{Mpc})$, independent of $a$.

It turns out to be sufficiently accurate to use the small- $x$ limit for $x \leqslant 3$ and the large- $x$ limit for $x \geqslant 9$. In the overlap region, $3 \leqslant x \leqslant 9$, results can be obtained that are $\pm 50 \%$ accurate by using whichever formula gives the smallest time. However, I have integrated numerically the equation

$$
\int_{1 / 2 x}^{x} \mathrm{~d} x x \Phi(x) \equiv x^{2} F(x)=K \tau .
$$

The results, accurate to $\pm 10 \%$, are given in the appendix, and have been used to generate Table I.

The table indicates significant contraction interior to two core radii. However, there are two effects that would tend to increase the times. First, I have assumed circular orbits, while the typical orbits are probably highly elliptical. I have not, as yet, worked out the numbers for elliptical orbits, but I think including this effect would tend to shorten the times interior to the core and lengthen them exterior to the core. Second, I have assumed a galactic mass of $10^{12} M_{\odot}$, which implies a radius (for an isothermal galaxy) of $100 \mathrm{kpc}$. As, in the center of the cluster, there is not room for

TABLE I

Times to reduce the radius to $\frac{1}{2}$ its initial value for two choices of the core radius of the Coma Cluster

\begin{tabular}{llll}
\hline $\begin{array}{l}R \\
(\operatorname{arcmin})\end{array}$ & $\begin{array}{l}R \\
(\mathrm{Mpc})^{\mathrm{a}}\end{array}$ & $\begin{array}{l}t_{1 / 2} \\
\left(r_{\mathrm{c}}=0.25 \mathrm{Mpc}\right)\end{array}$ & $\begin{array}{l}t_{1 / 2} \\
\left(r_{\mathrm{c}}=0.50 \mathrm{Mpc}\right)\end{array}$ \\
\hline 2.5 & 0.1 & $9.0 \times 10^{7} \mathrm{yr}$ & $4.8 \times 10^{7} \mathrm{yr}$ \\
5.0 & 0.2 & $6.8 \times 10^{8}$ & $3.6 \times 10^{8}$ \\
7.5 & 0.3 & $2.1 \times 10^{9}$ & $1.2 \times 10^{9}$ \\
10.0 & 0.4 & $4.5 \times 10^{9}$ & $2.7 \times 10^{9}$ \\
12.5 & 0.5 & $8.0 \times 10^{9}$ & $5.1 \times 10^{9}$ \\
15.0 & 0.6 & $1.3 \times 10^{10}$ & $8.4 \times 10^{9}$ \\
& & & \\
20.0 & 0.8 & $2.5 \times 10^{10}$ & $1.8 \times 10^{10}$ \\
25.0 & 1.0 & $4.2 \times 10^{10}$ & $3.2 \times 10^{10}$ \\
30.0 & 1.2 & $6.3 \times 10^{10}$ & $5.0 \times 10^{10}$ \\
& & & $1.0 \times 10^{11}$ \\
40.0 & 1.6 & $1.1 \times 10^{11}$ & $1.7 \times 10^{11}$ \\
50.0 & 2.0 & $1.8 \times 10^{11}$ & \\
\hline a Assumes & $1 \mathrm{arcmin}=40 \mathrm{kpc}$ &
\end{tabular}


$100-\mathrm{kpc}$ galaxies, the mass there should be reduced by at least a factor of 2 , which doubles the lifetimes.

Dynamical friction leads to mass segregation, since time scales inversely with mass. Bahcall (1973) found, by use of galaxy counts, core radii for Coma of $0.25 \mathrm{Mpc}$ $( \pm 20 \%$ ) for three limiting galaxy brightness's that differed by about 3 magnitudes. This result implies, assuming constant $M / L$, only modest mass segregation.

Oemler (1973) found the curious result that the space density had a hollow (a dip followed by a rise followed by a steady decrease) at about $1.2 \mathrm{Mpc}$. Could it be that the galaxies interior to $1.2 \mathrm{Mpc}$ spiralled in faster than those exterior to $1.2 \mathrm{Mpc}$ ?

I remind you that the spiralling in by dynamical friction does not rest on the more speculative scenario of torn-off halos. It is just another way of estimating the time scale for mass segregation in a self-gravitating system with a spectrum of masses. As Bahcall (1973) suggested, core radii of clusters of galaxies could be useful cosmological distance indicators. It is therefore of some importance to determine whether they evolve significantly in cosmological times.

I hope that I have convinced you that the parameters for the Coma Cluster justify an $N$-body simulation of this interesting object.

\section{Appendix}

The functions $\Phi(x)$ (defined by Equation (10)) and $F(x)$ (defined by Equation (11)) are presented below. $\Phi(x)$ has been calculated from tables of the isothermal sphere by Chandrasekhar and Wares (1949) and is accurate to the last digit. $F(x)$ was obtained by a three-point Simpson's Rule integration.

\begin{tabular}{rllllll}
\hline$x$ & $\Phi(x)$ & $F(x)$ & & $x$ & $\Phi(x)$ & $F(x)$ \\
\hline 1 & 0.7458 & 0.22 & & 14 & 3.4047 & 1.23 \\
2 & 1.3770 & 0.42 & & 15 & 3.4157 & 1.24 \\
3 & 1.8673 & 0.58 & & & \\
4 & 2.2424 & 0.71 & 16 & 3.4202 & 1.25 \\
5 & 2.5321 & 0.82 & 17 & 3.4194 & 1.26 \\
& & & 18 & 3.4144 & 1.27 \\
6 & 2.7575 & 0.91 & 19 & 3.4061 & 1.27 \\
7 & 2.9331 & 0.98 & 20 & 3.3953 & 1.27 \\
8 & 3.0693 & 1.04 & & & \\
9 & 3.1740 & 1.08 & & 21 & 3.3825 & 1.27 \\
10 & 3.2534 & 1.13 & 22 & 3.3682 & 1.27 \\
& & & 23 & 3.3528 & 1.27 \\
11 & 3.3126 & 1.16 & 24 & 3.3366 & 1.27 \\
12 & 3.3554 & 1.19 & 25 & 3.3199 & 1.27 \\
13 & 3.3853 & 1.21 & & & \\
\hline
\end{tabular}

\section{References}

Alladin, S. M.: 1965, Astrophys. J. 141, 768.

Alladin, S. M., Sastry, K. S., and Ballabh, G. M.: 1974, in B. Barbanis and J. Hadjidemetriou (eds.), Galaxies and relativistic Astrophysics, Springer-Verlag, Berlin, p. 129.

Alladin, S. M.. Potdar, A., and Sastry, K. S.: 1974. this volume, p. 167. 
Bahcall, N. A.: 1973, Astrophys. J. 183, 783.

Bardeen, J. M.: 1974, this volume, p. 297.

Biermann, P.: 1974, 'Report to the Faculty of Mathematical Sciences', George August University, Gottingen.

Chandrasekhar, S. and Wares, G. W.: 1949, Astrophys. J. 109, 551.

Gallagher, J. S. III and Ostriker, J. P.: 1972, Astron. J. 77, 288.

Hénon, M.: 1973, in L. Martinet and M. Mayor (eds.), Dynamical Structure and Evolution of Stellar Systems, Geneva Observatory, p. 214.

Lauberts, A.: 1974, Astron. Astrophys. 33, 231.

Oemler, A., Jr.: 1973, 'The Systematic Properties of Clusters of Galaxies', California Institute of Technology (Ph.D. Thesis).

Omer, G. C., Jr., Page, T. L., and Wilson, A. G.: 1965, Astron. J. 70, 440.

Ostriker, J. P. and Peebles, P. J. E. : 1973, Astrophys. J. 186, 467.

Peebles, P. J. E.: 1970, Astron. J. 75, 13.

Roberts, M. S.: this volume, p. 331.

Rood, H. J., Page, T. L., Kintner, E. C., and King, I. R.: 1972, Astrophys. J. 175, 627.

Saslaw, W. C. and de Young, D. S.: 1971, Astrophys. J. 170, 423.

Sastry, K. S. and Alladin, S. M.: 1970, Astrophys. Space Sci. 9, 261.

Spitzer, L., Jr.: 1969, Astrophys. J. 158, L139.

\section{DISCUSSION}

Miller: If you were to observe the cluster after this process, and use the virial theorem to estimate the mass, what would you get?

Lecar: The virial theorem would underestimate the mass of the cluster by the usual amount, because in this model, most of the mass of the cluster is in low luminosity halo material.

Hénon: It seems to me that this last argument of your students amounts in fact to considering only encounters between the galaxies and neglecting encounters between the galaxies and the hidden mass. So this might explain why you don't have dynamical friction and spiraling anymore. You still have encounters between galaxies in order to achieve hydrostatic equilibrium.

Lecar: In the problem considered by my students, the test galaxies were assumed to have negligible mass compared to the unseen (unspecified) material providing the gravitational field. This, admittedly, is an unrealistic dynamical model, but serves to illustrate the variety of solutions that are consistent with Poisson's equation and the equation of hydrostatic equilibrium (without, however, specifying how hydrostatic equilibrium is attained).

Gott: The rate at which a galaxy of mass $M_{g}$ spirals in due to dynamical friction is similar to the 2-body relaxation time calculated imagining the cluster mass to be composed completely of galaxies of mass $M_{g}$. Thus as in two body relaxation one finds that the most massive galaxies spiral toward the center most rapidly.

Lecar: Not quite. If the field galaxies and the test galaxy had the same mass, on the average, the test galaxy would not lose energy (although it's direction in space would be randomized). In the model I'm considering, the test galaxy is much more massive than the field stars and loses energy almost in every encounter.

Pișmiș: I like to make a comment: on observational grounds, it is shown independently by Page and Poveda that the percentage of the missing mass in systems of stars, or galaxies, is related to the total mass of the system in the sense that the larger the total mass of the system the larger is the percentage of the missing mass. I suspect your treatment would not take care of this property.

Saslaw: Do you think this process could account for the mass discrepancy claimed for small groups of galaxies?

Lecar: I don't think this (that is ripping off halos) would work for small groups of galaxies. 